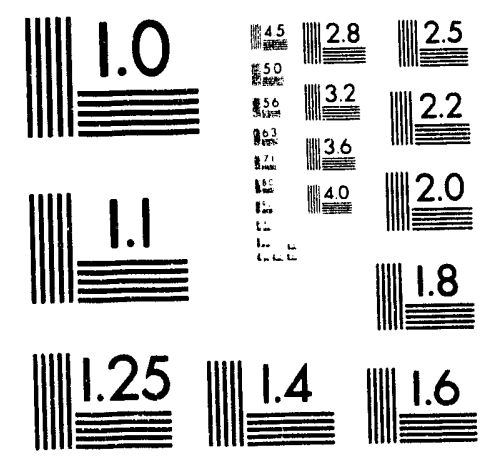



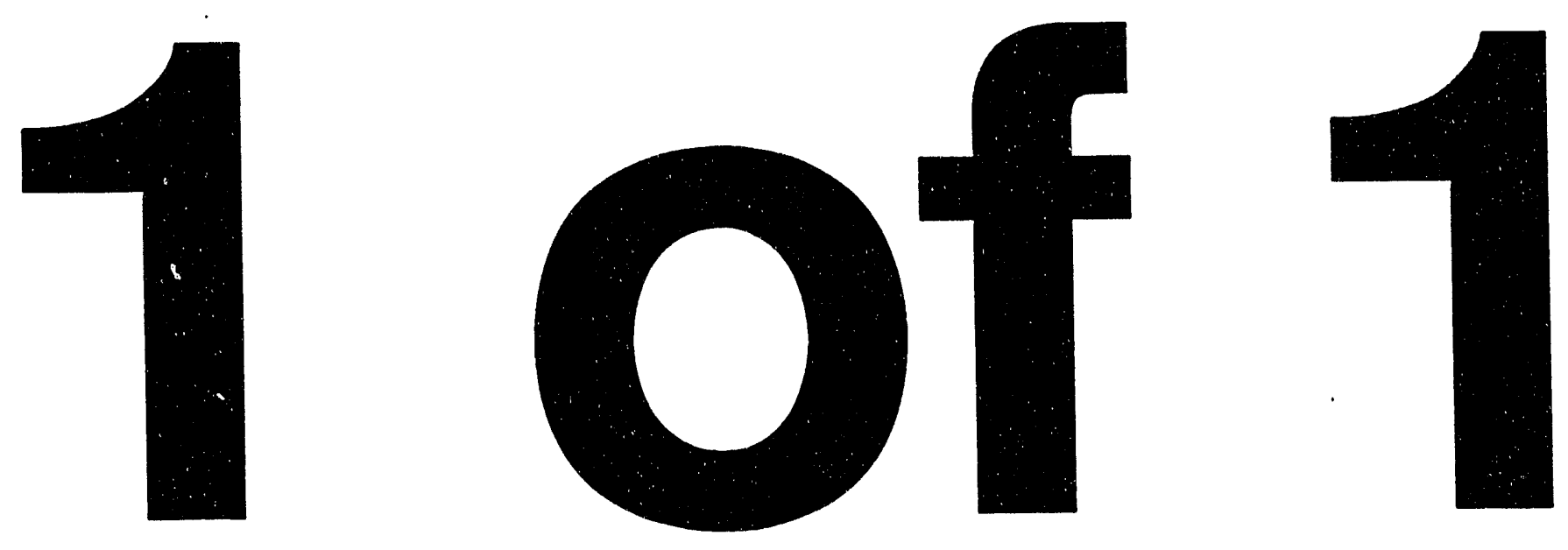


\title{
PERSISTENT WAKEFIELDS ASSOCIATED WITH WAVEGUIDE DAMPING OF HIGHER ORDER MODES*
}

\author{
Norman M. Kroll and Xintian Lin \\ University of California, San Diego, La Jolla, CA 92093 \\ Stanford Linear Accelerator Center, Stanford University, Stanford, CA 94309
}

\section{Abstract}

By means of an analytic model and numerical simulations we demonstrate that the wake of a waveguide damped cavity contains a persistent component which decays as $t^{-3 / 2}$ and thus is dominant at sufficiently large values of $t$. Because there are scenarios for which the effect gives rise to unacceptable beam instability, it cannot be ignored, but it seems likely that such an outcome can be avoided with proper design.

\section{Introduction}

The passage of a bunch of charged particles through an accelerator cavity generates a wakefield which may have a deleterious effect on the motion of subsequent bunches which pass through the cavity. The effect is often cumulative from bunch to bunch and from cavity to cavity and may lead to beam breakup [1]. One solution to this problem which has received extensive study is to drain the wakefield energy out of the cavity by means of waveguides coupled to the cavity and feeding into matched terminations [2]. The effectiveness of this procedure has typically been assessed by evaluating the resultant $Q_{\text {ext }}$ of higher order cavity modes, thereby determining their exponential damping rate. The purpose of this paper is to point out the existence of an additional effect which leads to a wakefield which falls off as $t^{-3 / 2}$ rather than exponentially. Because this effect always dominates at sufficiently long times, we refer to it as the persistent wake. It consists of a superposition of the frequencies of the various waveguide cutoff frequencies and may be understood as arising physically from the fact that the group velocity of each waveguide mode vanishes at its cutoff frequency. As we shall see below, for sulficiently heavy damping, it may dominate before the arrival of the second bunch. A similar phenomenon with essentially the same physical origin which occurs in the decay of unstable quantum states has received extensive study.

\section{The Dielectric Cavity Model}

As a simple model of a cavity heavily damped by waveguides, we consider a waveguide which has a finite section of length $h$ filled with dielectric. The configuration is illustrated in Fig. 1.

For brevity, we confine our attention to fields whose transverse configuration is that of the $\mathrm{TE}_{10}$ waveguide mode. There is always at least one trapped cavity mode in the dielectric, symmetric with respect to the $y-z$ plane,

- Work supported by U.S. Department of Energy grant DE-FG0392 ER40759 and contract DE-AC03-76SF00515

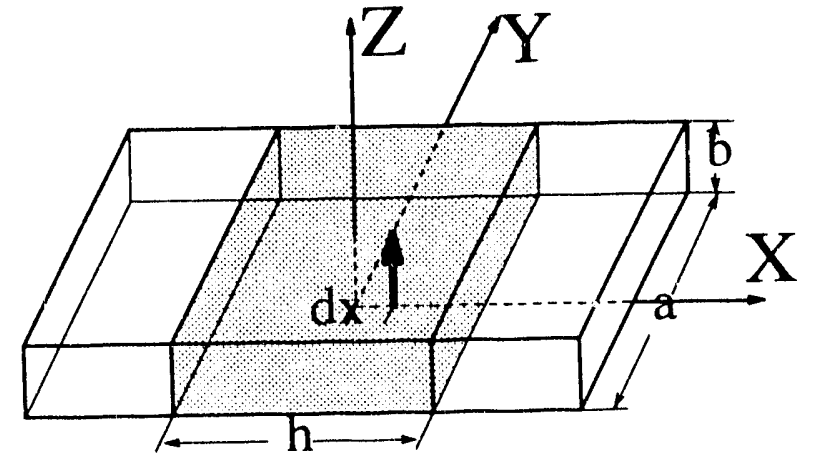

Figure 1: Dielectric cavity model

and with resonant frequency below the cutoff frequency of the dielectric free portion of the waveguide. This mode has an electric field distribution suitable for accelerating particles in the $\mathrm{z}$ direction along the $\mathrm{z}$ axis. There are also damped modes associated with multiple reflections between the two dielectric-free space interfaces which have frequencies above the cutoff of the free space portion of the waveguide. Hence it provides us with a reasonable model of a waveguide damped accelerator cavity.

To further simplify the problem we look only at the on axis transverse deflecting field $B_{y}$ generated by a unit charge moving relativistically in the $z$ direction and displaced by the infinitesimal distance $d_{x}$ from the $z$ axis, as shown in Fig. (1). Defining the transverse wake function $W_{\perp}$ as $B_{y} / d_{x}$ evaluated on the $z$ axis, and solving Maxwell's equations for our configuration we find

$$
\begin{gathered}
W_{\perp}=\int \frac{2}{a b}\left(e^{\frac{i \omega b}{c}}-1\right) \frac{k D_{2}}{\omega D_{1}} e^{-i \omega t} d \omega \\
D_{1}=\left(1+\frac{1}{\mu} \frac{k}{l}\right)-\left(1-\frac{1}{\mu} \frac{k}{l}\right) e^{i k h}, l=\frac{1}{c} \sqrt{\omega^{2}-\omega_{c}^{2}} \\
D_{2}=\left(1+\frac{1}{\mu} \frac{k}{l}\right)+\left(1-\frac{1}{\mu} \frac{k}{l}\right) e^{i k h}, k=\frac{1}{c} \sqrt{\epsilon \mu \omega^{2}-\omega_{c}^{2}}
\end{gathered}
$$

The integrand of Eq. (1) has a pair of branch points where $l$ vanishes, corresponding to the cutoff frequency in the dielectric free waveguides, and the Riemann surface of the integrand therefore has two sheets. In addition to the branch points the integrand has poles where $D_{1}$ vanishes. Causality and time reversal invariance tell us that we can choose the sign of $l$ so that there are no complex singularities in the complex plane cut along the real axis to the left and right of the branch points and extending to plus and minus infinity. These specifications define what is referred to as the physical sheet of the Riemann surface. We choose the parameters of the cavity so that there are no 
trapped deflecting nodes in order that we may expect $W_{\perp}$ to decay rapidly after the passage of the charge. Hence the only singularities on the physical sheet are the two branch points. The integral in Eq. (1) is taken along the entire real axis of the physical sheet with infinitesimal detours above them.

The second sheet of the Riemann surface is reached by crossing the branch cuts from the physical sheet. It has an infinite number of complex poles. These occur in groups of four, symmetrically distributed with respect to both axes. There may also be pairs of real poles between the branch points.

After the particle leaves the cavity $(t>b / c)$, the integral over the semi-circle at infinity in the lower half plane vanishes. We use it to close the integration path of Eq. (1) and shrink it to the form shown in Fig. (2). Here the upper half plane and the region between the two indicated branch cuts is a part of the physical sheet, while the remainder of the lower half plane is on the second sheet. In shrinking the contour integral to the two discontinuity integrals along the branch cuts, we must pass through the poles which have been exposed on the second sheet, and we must retain a residue from each of them. Thus we have expressed $W_{\perp}$ as an expansion in terms of the discrete exponentially damped cavity modes plus a contribution from the branch cut integrals. It is this latter contribution which provides the persistent wake.

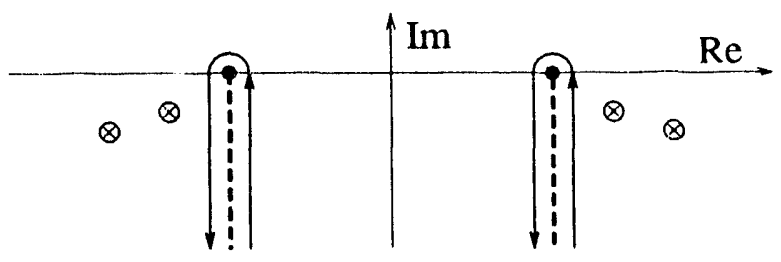

Figure 2: Contour for Calculating $W_{\perp}$

We define the persistent wake furction $W_{p}$ as the contribution from the branch cut discontinuity integrals, and to simplify the discussion we redefine $t$ as $\omega_{c} t$ and $\omega$ as $\omega / \omega_{c}$. The cut discontinuity can then be written as $\sqrt{\omega^{2}-1} F(\omega)$ where $F$ is analytic in the neighborhood of the branch point. Making use of partial integration, we obtain the large $t$ asymptotic behaviour as follows:

$$
\begin{aligned}
W_{p}(t) & =\int_{1}^{1-i \infty} \sqrt{\omega^{2}-1} F(\omega) e^{-i \omega t} d \omega \\
& =\frac{1}{i t} \int_{1}^{1-i \infty} e^{-i \omega t} d \omega \frac{d}{d \omega} \sqrt{\omega^{2}-1} F(\omega) d \omega \\
& =\frac{1}{i t} \int_{1}^{1-i \infty} e^{-i \omega t} \frac{F(1)}{\sqrt{\omega^{2}-1}} d \omega+ \\
& \frac{1}{i t} \int_{1}^{1-i \infty} e^{-i \omega t} \sqrt{\omega^{2}-1} G(\omega) d \omega+c . c .
\end{aligned}
$$

Here $G$, which has the same analyticity properties as $F$, is given by

$$
G(\omega)=\frac{F(\omega) \omega-F(1)}{\omega^{2}-1}+\frac{d F}{d \omega}
$$

The dominant asymptotic behavior comes from the first term. Carrying out the integral we find

$$
W_{p}(t)=-\frac{\pi}{2 t} H_{0}^{(2)}(t) F(1)+c . c .
$$

which decays as $t^{-3 / 2}$. Here $H_{0}^{(2)}$ is the zeroth order Hankel function of the second kind. Because the second term is subject to the same manipulations as the first, it falls off as $t^{-5 / 2}$, and by repeating the process one can get. a sequence terms falling off with increasing powers of $1 / t$.

The full expression for $W_{\perp}$ has been evaluated numerically. Highly damped successive reflections from the interfaces appear at early time, but as shown in Fig. (4) the asymptotic limit takes over quite rapidly.

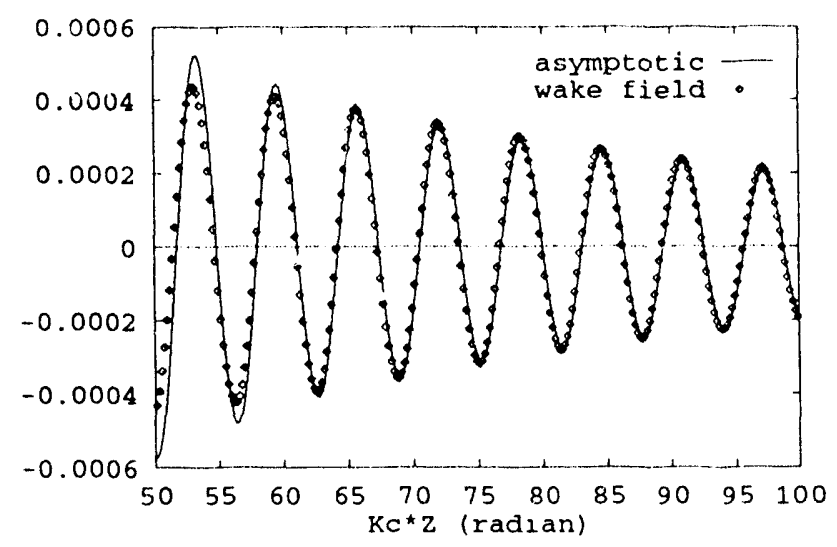

Figure 3: Asymptotic behavior of the wake field

\section{2-D simulation}

For our simulation structure we have taken a cavity with four damping waveguides with the cross section shown in Fig. (4a). The scale is chosen so that the cavity has an accelerating mode at $11.424 \mathrm{GHz}$. The waveguide cutoff for the lowest mode is $14.27 \mathrm{GHz}$. 'Two damped dipole modes were identified with MAFIA frequency domain calculations. Their frequencies, 17.365 and $34.297 \mathrm{GHz}$, and Q's, 3.74 and 9.0 were determined using Kroll-Yu [3] and Kroll-Lin [4] for the former, and because the latter was above cutoff for two waveguide modes, Kroll-Kim-Yu [5] was required. A MAFIA time domain computation determined the wakefield excited by a Gaussian line charge bunch displaced from the origin. The wakefield obtained for a $\sigma_{z}$ of $2.6 \mathrm{~mm}$ is described by Figs. ( $\left.4 \mathrm{~b}, \mathrm{c}, \mathrm{d}\right)$. The abscissa represents the distance of a synchronous trailing bunch behind the excitation bunch, a typical value being $42 \mathrm{~cm}$. Fig. (4b) shows the persistent wake dominating after $25 \mathrm{~cm}$. The logarithmic plot of the averaged amplitude shown in Fig (4c) exhibits perhaps three exponential damping rates. The straight line fit shown determines a damping rate of $44.33 \mathrm{~m}^{-1}$. This is to be compared with the unresolved pair of decay rates of $48.64 \mathrm{~m}^{-1}$ and 39.92 $m^{-1}$ from the first and second dipole modes. The second 


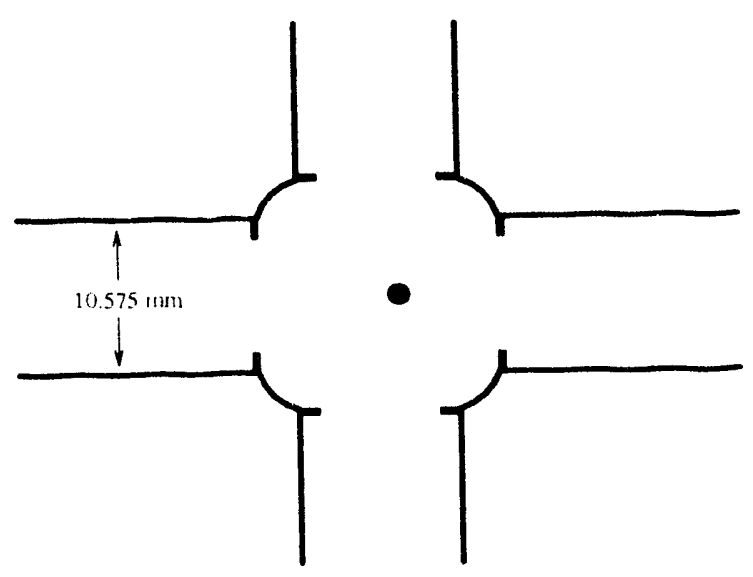

(a)

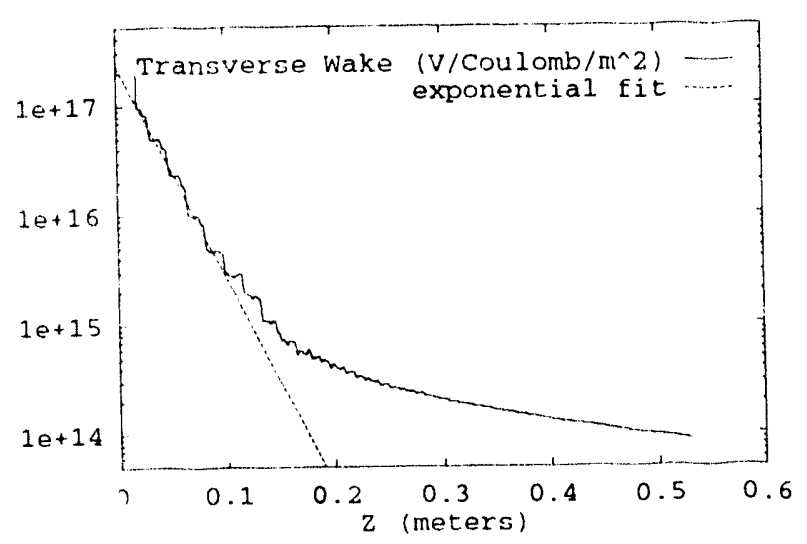

(c)

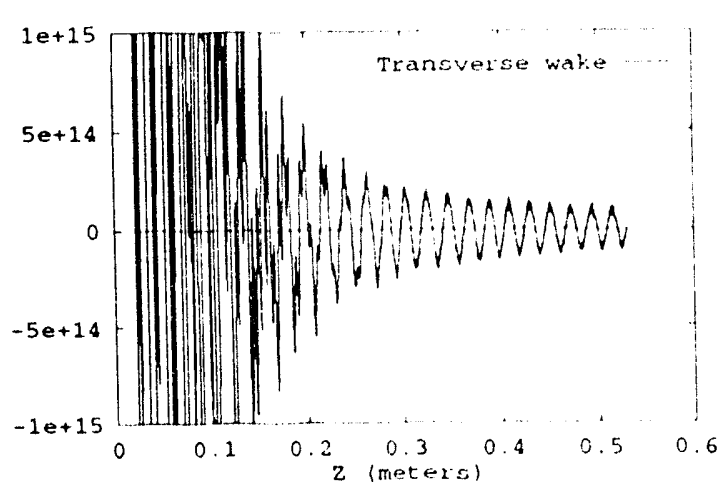

(b)

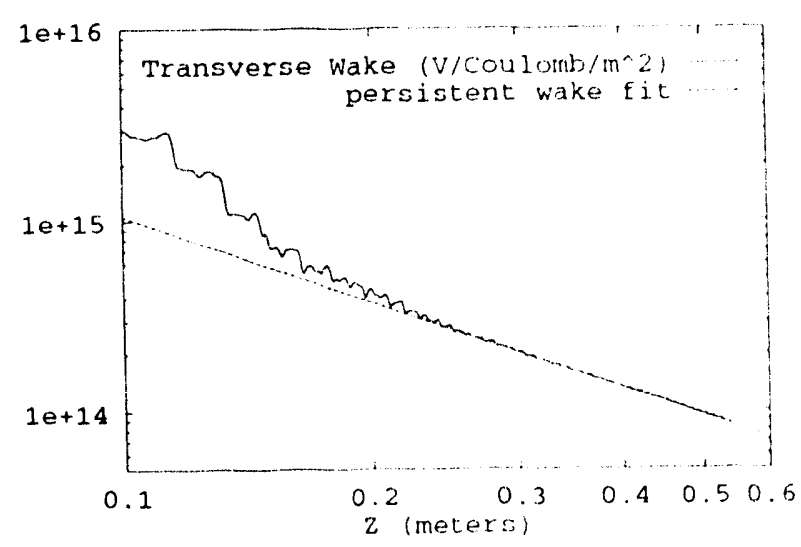

(d)

Figure 4: (a): 2I) strongly damped structure, the dot refers to beam path in third dimension. (b): Dipole wake field as a function of distance $=t * c$. (c): $\log$ plot of the same wake field, dashed line refers to the best exproncential fit. (d): $\log -\log$ plot, dashed line is $t^{-3 / 2}$

slope of $27.6 \mathrm{~m}^{-1}$ (straight line is not shown) was associated with a high frequency mode beyond the range of our frequency domain simulation. The log-log plot of Fig (4.2d) compares the persistent wake with a $t^{-3 / 2}$ straight line.

\section{Concluding Remarks}

The magnitude of the wake at $42 \mathrm{~cm}$ appears to be large cnough to cause some beam blow up. We have also studied the extreme case of the cavity formed by two waveguides intersecting at right angles. While no damped dipole resonances could be idnutified, indicating extreme higher order mode damping, the persistent wake was four times as large, and the beam blow up would be disastrous. 'This suggests that an iris which decreases the damping tends to decouple the cavity from the persistent wake, and hence that better results could bo obtained by further decreasing the damping. Detuning the persistent wake by distributing the waveguide cutoff frequencies, which should be simpler than detuning the HOM's, would also be expected to be effective. It is also likely that the effect would be negligible in the choke mode cavity damping scheme [6].

\section{References}

[1] K. Thompson and R. Ruth, Phys Rev D 41, 964 (1990)

[2] R. Palmer, High Energy Physics an the 1990's, S. Jenson, Ed. (World Scientific, Singapore, 1989), plo.fi38641

[3] N. Kroll and D. Yu, Part. Accel. 34, 2:31 (1990)

[4] N. Kroll and X. Lin, Proc. 1990 Linac Conf., Alluquerque, NM, June 10-14 (19990) p. 2:3x

[5] N. Kroll, J. Kin, and 1). Yu Pror. lg9: linar Conf. Ottawa, Ontario, August, 24-28 (1992) Al:(:1-10728 $217(1992)$

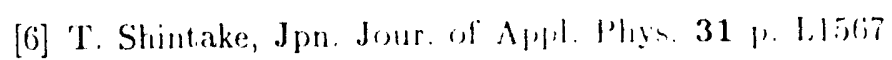
$(1992)$ 

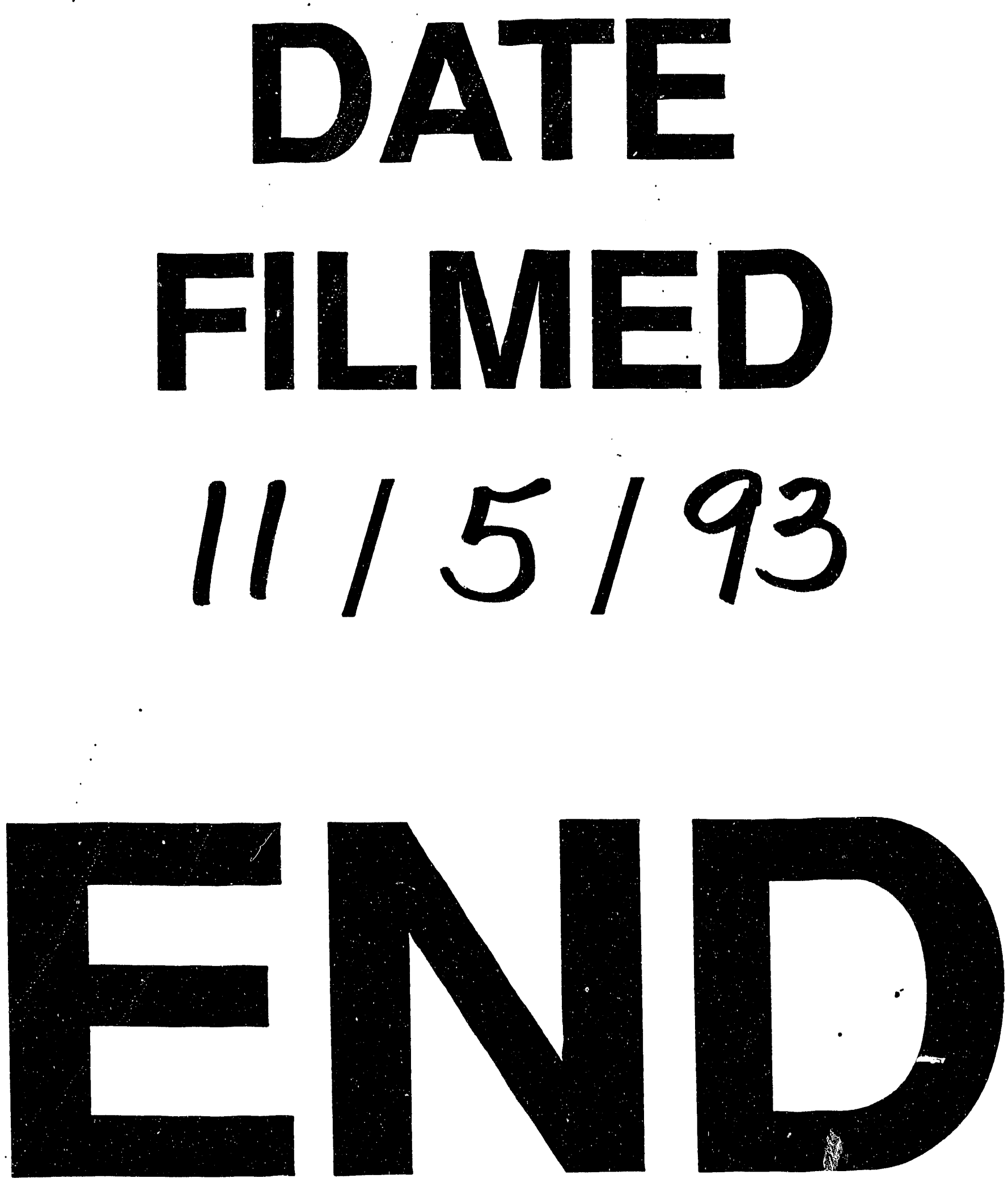
